\title{
Marker Assisted Selection in Comparison to Conventional Plant Breeding: Review Article
}

\author{
Melese Lema* \\ Assistant Crop Improvement Researcher At Southern Agricultural Research Institute, Ethiopia
}

Submission: January 18, 2018; Published: February 28, 2018

"Corresponding author: Melese Lema, Assistant Crop Improvement Researcher At Southern Agricultural Research Institute, PO Box 06, Hawassa, Ethiopia, Email: meleselema72@gmail.com

\begin{abstract}
Although significant strides have been made in crop improvement through phenotypic selections for agronomical important traits, considerable difficulties are often encountered during this process. A new variety in conventional breeding could take 8 to 10 years to develop. Breeders are very interested in new technologies to speed up this process or make it more efficient. The development of molecular markers was therefore greeted with great enthusiasm as it was seen as a major breakthrough promising to overcome this key limitation. Marker assisted selection is likely to become more valuable as a larger number of genes are identified and their functions and interactions elucidated; Reduced costs and optimized strategies for integrating MAS with phenotypic selection are needed before the technology can reach its full potential. Overall, marker assisted selection has proven to be a very useful technique in plant breeding. Through these techniques, plant breeders have been able to produce cultivars of agriculturally significant plants with genes for resistance to many diseases that were not possible before the advent of DNA technology.
\end{abstract}

\section{Introduction}

Plant breeding is the art and science of changing the traits of plants in order to produce desired characteristics and it can be accomplished through many different techniques ranging from simply selecting plants with desirable characteristics for propagation, to methods that make use of knowledge of genetics and chromosomes, to more complex molecular techniques.

Conventional plant breeding is primarily based on phenotypic selection of superior individuals among segregating progenies resulting from hybridization. Although significant strides have been made in crop improvement through phenotypic selections for agronomical important traits, considerable difficulties are often encountered during this process, primarily due to genotype - environment interactions. Besides, testing procedures may be many times difficult, unreliable or expensive due to the nature of the target traits (e.g. abiotic stresses) or the target environment [1]. A new variety in conventional breeding could take 8 to 10 years to develop. Breeders are very interested in new technologies to speed up this process or make it more efficient.

The development of molecular markers was therefore greeted with great enthusiasm as it was seen as a major breakthrough promising to overcome this key limitation. With the advent of DNA-based genetic markers, it became possible to identify large numbers of markers dispersed throughout the genetic material of any species of interest and use the markers to detect associations with traits of interest John \& Andrea [2], thus allowing marker assisted selection (MAS) finally to become a reality.

Molecular marker-assisted selection, often simply referred to as marker-assisted selection (MAS) involves selection of plants carrying genomic regions that are involved in the expression of traits of interest through molecular markers. With the development and availability of an array of molecular markers and dense molecular genetic maps in crop plants, MAS has become possible for traits both governed by major genes as well as quantitative trait loci (QTLs).

The potential benefits of using markers linked to genes of interest in breeding programmes, thus moving from phenotype based towards genotype-based selection, have been obvious for many decades. By now a stage has been reached, where genomics research is focusing on generating functional markers that can help identifying genes that underlie certain traits, thus facilitating their exploitation in crop improvement programs.

The mapping of genes controlling agronomic traits coupled with the widespread availability of easy to use simple sequence repeat (SSR) markers and quick DNA extraction methods has provided breeders with an excellent opportunity to apply marker assisted selection (MAS) methods in varies of crops David 2007. 
The objective of this review is to provide an overview of the requirements for conducting a MAS program in crop plants, discuss the breeding strategy for MAS, and also some of the advantages and drawbacks of MAS in comparison to conventional breeding (Table 1).

Table 1: Examples of gene or QTL pyramiding in cereals Bertrand(2008).

\begin{tabular}{|c|c|c|c|c|c|}
\hline Species & Trait(s) & Genes from parent1 & Genes from parent2 & $\begin{array}{l}\text { Section } \\
\text { stage }\end{array}$ & Section stage \\
\hline barley & $\begin{array}{l}\text { barley yellow } \\
\text { Mosaic virus }\end{array}$ & rym 1 & rym5 & $\mathrm{F}_{2}$ & RFLP,CAPS \\
\hline barley & $\begin{array}{l}\text { barley yellow } \\
\text { Mosaic virus }\end{array}$ & rym4,rym9,rym11 & rym4,rym9,rym11 & $\begin{array}{c}\mathrm{F}_{1}- \\
\text { derived } \\
\text { doubled } \\
\text { haploids }\end{array}$ & RAPD,SSR \\
\hline barley & stripe rust & $\begin{array}{l}\text { Rspx } \\
\text { Rspx }\end{array}$ & $\begin{array}{c}\text { QTLs 4,7 } \\
\text { QTL } 5\end{array}$ & $\begin{array}{c}\mathrm{F}_{1}^{-} \\
\text {derived } \\
\text { doubled } \\
\text { haploids }\end{array}$ & SSR \\
\hline rice & bacterial blight & xa5,ха13 & Xa4,Xa21 & $\mathrm{F}_{2}$ & RFLP,STS \\
\hline rice & $\begin{array}{l}\text { bacterialblight,yellow } \\
\text { stem borer,sheath blight }\end{array}$ & $\begin{array}{l}\text { bacterialblight,yellow stem } \\
\text { borer,sheath blight }\end{array}$ & $\mathrm{RC} 7$ chitinase gene, $\mathrm{Bt}$ & $\mathrm{F}_{2}$ & STS \\
\hline rice & blast disease & Pil,piz-5 & Pil,Pita & $\mathrm{F}_{2}$ & RFLP,STS \\
\hline rice & $\begin{array}{l}\text { insect resistance and } \\
\text { bacterial blight }\end{array}$ & Xa21 & bt & $\mathrm{F}_{2}$ & STS \\
\hline wheat & powdery mildew & $\mathrm{Pm} 2$ & $\operatorname{Pm} 4 a$ & $\mathrm{~F}_{2}$ & RLFP \\
\hline
\end{tabular}

Breeding strategies in marker-assisted selection compared to conventional selection

The breeding strategies for which MAS is used most frequently, are selection of simple traits or QTLs from breeding lines/populations, introgression of genes from breeding lines or wild relatives, MABC, marker-assisted recurrent selection (MARS), and pyramiding of genes.

Marker-assisted evaluation of breeding material compared to conventional evaluation

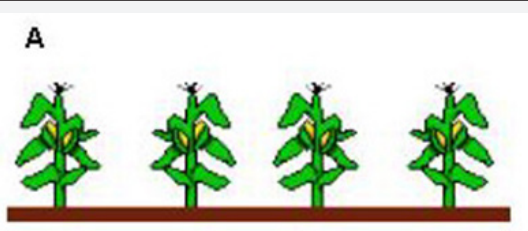

B

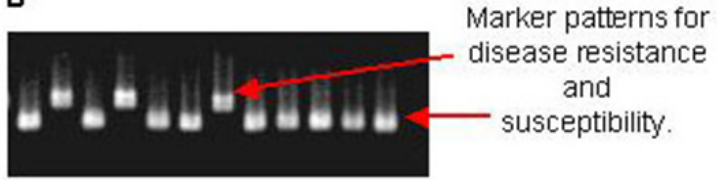

Figure 1: conventional selection (A) In marker-assisted selection (B)

Prior to crossing (hybridization) and line development, there are several applications in which DNA marker data may be useful for breeding, such as cultivar identity, assessment of genetic diversity and parent selection, and confirmation of hybrids. Traditionally, these tasks have been done based on visual selection and analyzing data based on morphological characteristics (Figure 1).

Cultivar identity/assessment of purity: In practice, seed of different strains is often mixed due to the difficulties of handling large numbers of seed samples used within and between crop breeding programmes. Markers can be used to confirm the true identity of individual plants. The maintenance of high levels of genetic purity is essential in cereal hybrid production in order to exploit heterosis. In hybrid rice, SSR and STS markers were used to confirm purity, which was considerably simpler than the standard 'grow-out tests' that involve growing the plant to maturity and assessing morphological and floral characteristics [3].

Assessment of genetic diversity and parental selection: Breeding programmes depend on a high level of genetic diversity for achieving progress from selection. Broadening the genetic base of core breeding material requires the identification of diverse strains for hybridization with elite cultivars [4,5]. Numerous studies investigating the assessment of genetic diversity within breeding material for practically all crops have been reported. DNA markers have been an indispensable tool for characterizing genetic resources and providing breeders with more detailed information to assist in selecting parents. In some cases, information regarding a specific locus (e.g. a specific resistance gene or QTL) within breeding material is highly desirable. For example, the comparison of marker haplotypes has enabled different sources of resistance to Fusarium head blight, which is a major disease of wheat worldwide, to be predicted $[6,7]$ (Figure 2). 


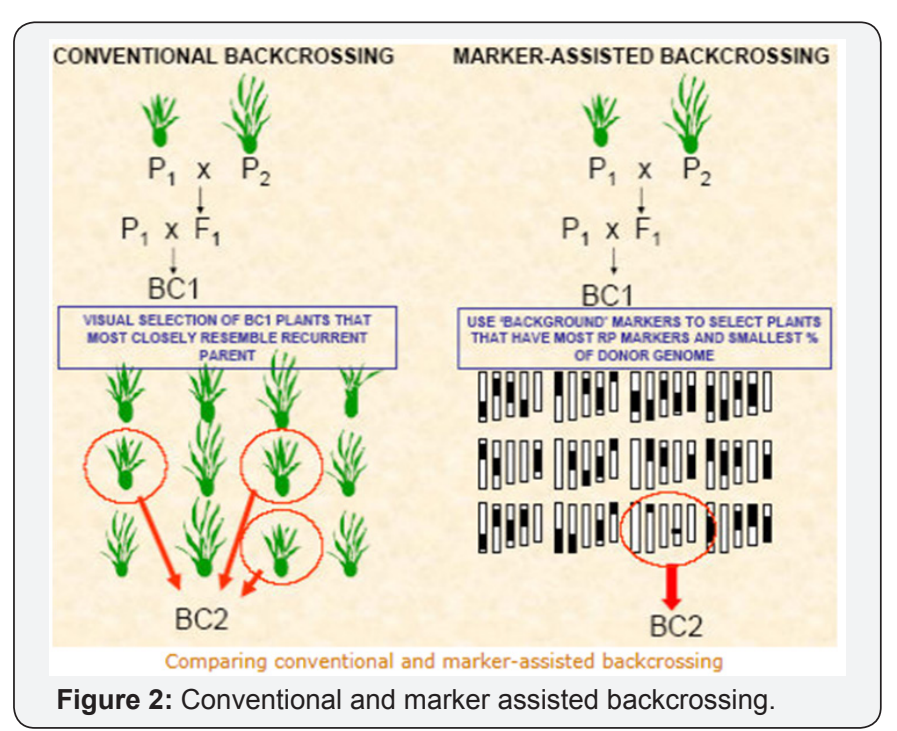

\section{Marker-assisted backcrossing (MABC) compared to conventional backcrossing}

Backcrossing is used in plant breeding to transfer (introgress) favorable traits from a donor plant into an elite genotype (recurrent parent). In repeated crossings the original cross is backcrossed with the recurrent parent until most of the genes stemming from the donor are eliminated. However, the donor segments attached to the target allele can remain relatively large, even after many backcrossing generations. In order to minimize this linkage drag, marker assays can be of advantage [8]. Markers can be used in the context of MABC to either control the target gene (foreground selection), to accelerate the reconstruction of the recurrent parent genotype (background selection) or to select backcross progeny with the target gene and recombination events between the target locus and linked flanking markers (recombinant selection). According to Tanksley et al. [9], in traditional backcross breeding the reconstruction of the recurrent parent genotype requires more than six generations, while this may be reduced to only three generations in MABC.

At the moment MABC also is and will probably remain the preferred means of backcrossing transgenes into elite inbred lines, which is also considerably contributing to its popularity [10]. MABC is especially efficient if a single allele is to be transferred into a different genetic background, for example, in order to improve an existing variety for a specific trait. However, if the performance of a plant is determined by a complex genotype it is unlikely that this ideal genotype will be attained through MABC only [11]. To overcome the limitation of only being able to improve existing elite genotypes, other approaches like markerassisted recurrent selection (MARS) have to be considered. In general, MAB will probably become an increasingly more popular approach, largely for the same reasons that conventional backcrossing has been widely used [12].

\section{Marker-assisted recurrent selection (MARS) Compared to conventional selection}

The improvement of complex traits via phenotypic recurrent selection is generally possible, but the long selection cycles impose restrictions on the practicability of this breeding method. With the use of markers, recurrent selection can be accelerated considerably. In continuous nursery programs pre-flowering genotypic information is used for marker-assisted selection and controlled pollination. Thus, several selection-cycles are possible within one year, accumulating favorable QTL alleles in the breeding population [13].

Additionally, it is possible today to define an ideal genotype as a pattern of QTLs, all QTLs carrying favorable alleles from various parents. If individuals are crossed based on their molecular marker genotypes, it might be possible to get close to the ideal genotype after several successive generations of crossings. It is likely that through such a MARS breeding scheme higher genetic gain will be achieved than through MABC [11]. Concepts how to achieve the ideal genotype using multi-trait selection indices have been developed [14].

\section{Marker-assisted pyramiding}

Using MAS, several genes can be combined into a single genotype. Pyramiding is also possible through conventional breeding but it is usually not easy to identify the plants containing more than one gene and phenotypically testing individual plants for all traits can be time-consuming and sometimes very difficult. The most frequent strategy of pyramiding is combining multiple resistance genes. Different resistance genes can be combined in order to develop broad-spectrum and durable resistance to, e.g., diseases and insects. The ability of a pathogen to overcome two or more effective genes by mutation is considered much lower compared with the 'conquering' of resistance controlled by a single gene. Therefore, it may be very difficult to assess plants from certain population types (e.g. F2) or for traits with destructive bioassays. DNA markers can greatly facilitate selection because DNA marker assays are non-destructive and markers for multiple specific genes can be tested using a single DNA sample without phenotyping.

The most widespread application for pyramiding has been for combining multiple disease resistance genes (i.e. combining qualitative resistance genes together into a single genotype). In order to pyramid disease or pest resistance genes that have similar phenotypic effects, and for which the matching races are often not available, MAS might even be the only practical method - especially where one gene masks the presence of other genes $[15,16]$. However, phenotypic selection may not be carried out due to the lack of differentiating virus strains. Thus, MAS offers promising opportunities. What has to be taken into account when applying such strategies in practical breeding is the fact that the pyramiding has to be repeated after each crossing, 
because the pyramided resistance genes are segregating in the progeny [17]. In the future, MAS pyramiding could also facilitate the combination of QTLs for abiotic stress tolerances, especially QTLs effective at different growth stages.

\section{Early generation marker-assisted selection compared to conventional selection}

Although markers can be used at any stage during a typical plant breeding programme, MAS is a great advantage in early generations because plants with undesirable gene combinations can be eliminated. This allows breeders to focus attention on a lesser number of high-priority lines in subsequent generations. When the linkage between the marker and the selected QTL is not very tight, the greatest efficiency of MAS is in early generations due to the increasing probability of the same phenotype, necessitating a progeny test to determine which plants possess more than one gene.

An important prerequisite for successful early-generation selection with MAS are large populations and low heritability of the selected traits, as under individual selection, the relative efficiency of MAS is greatest for characters with low heritability [18]. Results from Kuchel [19] and Bonnet et al. [20] show that maximum gain can be achieved at lowest cost in marker-assisted wheat breeding when molecular markers, closely linked to target genes, are utilized to enrich target loci within segregating populations in early generations.

The major disadvantage of applying MAS at early generations is the cost of genotyping a larger number of plants. One strategy proposed by Ribaut \& Betran [21] involving MAS at an early generation was called single large-scale MAS (SLS-MAS). The authors proposed that a single MAS step could be performed on F2 or F3 populations derived from elite parents. This approach used flanking markers (less than $5 \mathrm{CM}$, on both sides of a target locus) for up to three QTLs in a single MAS step. Ideally, these QTLs should account for the largest proportion of phenotypic variance and be stable in different environments.

\section{Combined marker-assisted selection}

There are several instances when phenotypic screening can be strategically combined with MAS. In the first instance, 'combined MAS' (coined by Moreau et al. [22]) may have advantages over phenotypic screening or MAS alone in order to maximize genetic gain Lande \& Thompson [18]. This approach could be adopted when additional QTLs controlling a trait remain unidentified or when a large number of QTLs need to be manipulated. Bohn et al. [23] investigated the prospect of MAS for improving insect resistance in tropical maize and found that MAS alone was less efficient than conventional phenotypic selection. However, there was a slight increase in relative efficiency when MAS and phenotypic screening were combined.

In an example in wheat, MAS combined with phenotypic screening was more effective than phenotypic screening alone for a major QTL on chromosome 3BS for Fusarium head blight resistance [24]. In practice, all MAS schemes will be used in the context of the overall breeding programme, and this will involve phenotypic selection at various stages. This will be necessary to confirm the results of MAS as well as select for traits or genes for which the map location is unknown. In some (possibly many) situations, there is a low level of recombination between a marker and QTL, unless markers flanking the QTL are used [15,25]. In other words, a marker assay may not predict phenotype with $100 \%$ reliability.

However, plant selection using such markers may still be useful for breeders in order to select a subset of plants using the markers to reduce the number of plants that need to be phenotypically evaluated. This may be particularly advantageous when the cost of marker genotyping is cheaper than phenotypic screening, such as for quality traits [26]. This was referred to as 'tandem selection' by Han et al. [26] and 'stepwise selection' by Langridge \& Chalmers [27]. In addition to complementing conventional breeding methods, mapping QTLs for important traits may have an indirect benefit in a conventional breeding program. In many cases, this occurs when traits which were thought to be under the complex genetic control are found to be under the influence of one or a few major QTLS.

\section{Application of markers in germplasm storage, evaluation and use}

Marker-assisted germplasm evaluation is an important tool in the acquisition, storage and use of plant genetic resources (PGR) Bretting \& Widrlechner [28] and the evaluation of germplasm can be considerably improved with the assistance of markers. Markers can be used prior to crossing to evaluate the breeding material. Also, mixing of seed samples can be discovered using markers instead of growing plants to maturity and assessing morphological characteristics [3]. In order to broaden the genetic base of core breeding material, germplasm of diverse genetic background for crossings with elite cultivars can be identified with the assistance of markers Xu et al. [4] and markers are on the whole a valuable tool for characterizing genetic resources, delivering detailed information usable in selecting parents. According to Xu et al. [4], molecular markers can be used for

a. Differentiating cultivars and creating, maintaining, and improving heterotic groups;

b. Assessing collections and identifying germplasm redundancy, underrepresented alleles, and genetic gaps;

c. Monitoring genetic shifts that can occur during medium- or long-term storage, regeneration, domestication, and breeding;

d. Identifying unique germplasm; and

e. Constructing core collections. 


\section{Advantages of marker-assisted breeding over conventional plant breeding}

The use of DNA markers for screening and selecting of plants in a breeding program provides several advantages and is therefore very attractive to plant breeders [29].

a. As DNA marker based genotypes can be obtained from almost any plant tissue, plants can be screened already at the seedling stage or even as seeds, thus allowing early selection for traits which may be expressed in adult plants only (i.e. grain or fruit quality, male sterility, photoperiod sensitivity). With the availability of pre flowering genotypic information MAS allows for controlled pollination, e.g. in marker-assisted recurrent selection.

b. Target alleles that are difficult, expensive and/or time consuming to score phenotypically can be selected with the assistance of markers (e.g. environmentally sensitive traits, as DNA markers are mostly neutral to environmental variation).

c. Selections can be made on a single plant basis where this would not be possible by phenotypic selection. Poor heritability does not pose a problem if selection is based on marker information.

d. For traits with complex inheritance every individual genetic component contributing to the trait can be selected separately. Also, multiple characters that would normally be epistatic (i.e. they show a certain positive or negative effect only in combination with each other) can be maintained and ultimately fixed.

e. Recessive genes can be maintained without the need for progeny tests in each generation, as homozygous and heterozygous plants can be distinguished with the aid of (codominant) markers. In backcrossing, DNA markers can help to minimize linkage drag around the target gene and reduce the generations required to recover a recurrent parent's genetic background

f. In the choice of parents in crossing programs markers can be applied too. Here they can either help to maximize diversity, and in this way support the exploitation of heterosis, or they can minimize diversity, if gene complexes built up in elite inbred germplasm are to be preserved.

Potential Drawbacks and Discrepancy in MAS Application Compared to Conventional Plant Breeding

\section{Potential drawbacks}

Still at the early stages of DNA marker technology development: Although DNA markers were first developed in the late 1980 s, more user-friendly PCR-based marker such as SSRs were not developed until the mid- to late 1990s. Although currently large numbers of SSRs are publicly available for major cereals, this number was initially very low. It is only during the last 5-7 years that these markers could have been widely used, and tangible results may not yet have been produced. If this is the case, there should be a notable increase in the number of published papers describing MAS in the next 10 years and beyond [29].

Marker-assisted selection results may not be published: Although QTL mapping has many potential practical outcomes, it is considered to be a basic research process, and results are typically published in scientific journals. However, for plant breeding, the final 'product' is a new variety. Although these varieties are registered, explicit details regarding the use of DNA markers during breeding may not be provided. Another reason for the limited number of published reports could be that private seed companies typically do not disclose details of methodology due to competition with other seed companies. In general, the problem of publishing also extends to QTL validation and QTL mapping. New QTLs are frequently reported in scientific journals, but reconfirmation of these QTLs in other germplasm and identification of more useful markers are usually not considered novel enough to warrant new publications [29].

\section{Reliability and accuracy of quantitative trait loci mapping studies}

The accuracy of the QTL mapping study is critical to the success of MAS. This is particularly important when QTL mapping is undertaken for more complex traits, such as yield, that are controlled by many QTLs with small effects compared with simple traits. Many factors may affect the accuracy of a QTL mapping study such as the level of replication used to generate phenotypic data and population size [30]. Simulation and experimental studies have indicated that the power of QTL detection is low with the typical populations (less than $200)$ that are used [30,31]. As a result, confidence intervals for regions containing QTLs may be large, even for QTLs with large effects. Furthermore, sampling bias can lead to a large bias in estimates of QTL effects, especially in relatively small population sizes [32]. These factors have important implications for MAS, since the basis for selecting markers depends on the accurate determination of the position and effect of a QTL.

\section{Insufficient linkage between marker and gene/ quantitative trait locus}

In some cases, recombination occurs between the marker and gene/QTL due to loose linkage [25]. This may occur even if genetic distances from a preliminary QTL mapping study indicated tight linkage, because data from a single QTL mapping experiment may not be accurate [25]. The process of marker validation is required to determine the reliability of a marker to predict phenotype and these points out the advantages of using flanking markers. 
Limited markers and limited polymorphism of markers in breeding material

Ideally, markers should be 'diagnostic' for traits in a wide range of breeding material. In other words, markers should clearly discriminate between varieties that do and do not express the trait. Unfortunately, in practice, DNA markers are not always diagnostic. For example, a wheat SSR marker was diagnostic for the Sr2 gene (controlling stem rust resistance) for all except four susceptible Australian cultivars, in which the same marker allele was detected as for the source of resistance. This would preclude the use of this SSR marker for the introgression of resistance in the four susceptible cultivars, requiring that additional markers be developed. Even with the large numbers of available markers in some crops, there can be specific chromosome regions containing an important gene or QTL for which it is difficult to find polymorphic markers [29].

\section{Effects of genetic background}

It has been observed that QTLs identified in a particular mapping population may not be effective in different backgrounds. For example, Steele et al. 2006 found that only one of four root length QTLs were effective when transferred by backcrossing into a new rice variety. In some cases, this is due to the small effect of an allele transferred into elite varieties. Often for QTL mapping experiments, parents that represent the extreme ends of a trait phenotype are selected. This increases the chance of detecting QTLs because QTL mapping is based on statistically different means of marker groups. The main disadvantage with this approach is that one (or even both) parent(s) may possess QTL alleles that are similar or even identical to the elite germplasm used in breeding programmes. In this case, the effect of a QTL may be insignificant when used for introgression into elite varieties. In other cases, the effect of a QTL may differ in different genetic backgrounds due to interactions with other loci or epistasis [33].

\section{Quantitative trait loci! Environment effects}

While the effects of many QTLs appear to be consistent across environments, the magnitude of effect and even direction of QTLs may vary depending on environmental conditions due to QTL environment interactions [33]. This often occurs for QTLs with smaller effects. The extent of QTL environment interactions is often unknown because the QTL mapping studies have been limited toonly a few years (replications) or locations. The existence of QTL environment interactions must be carefully considered in order to develop an effective MAS scheme [25].

\section{High cost of marker-assisted selection}

The cost of using MAS compared with conventional phenotypic selection may vary considerably, although only a relatively small number of studies have addressed this topic. Landmark papers by Dreher et al. and Morris et al. showed that the cost-benefit ratio of MAS will depend on several factors, such as the inheritance of the trait, the method of phenotypic evaluation, the cost of field and glasshouse trials and labor costs. It is also worth noting that large initial capital investments are required for the purchase of equipment, and regular expenses will be incurred for maintenance. Intellectual property rights, for example, licensing costs due to patents, may also affect the cost of MAS. One approach to this problem is to contract the marker work out to larger laboratories that can benefit from economies of scale and high throughput equipment.

\section{Application gap between research laboratories and plant breeding institutes}

In many cases, QTL mapping research is undertaken at universities whereas breeding is generally undertaken at different locations such as research stations or private companies. Consequently, there may be difficulties in the transfer of markers and relevant information to breeders in situations where the two groups do not work closely together. More importantly, Van Sanford et al. also pointed out that transfer problems may be related to the culture of the scientific community. Given the emphasis on conducting innovative research, and on the publication of research results within academic institutions, scientists do not have much motivation to ensure that markers are developed into breeder-friendly ones and that they are actually applied in breeding programmes. This is even truer for activities in the private sector where publication of results is generally discouraged [25].

\section{Knowledge gap among molecular biologists, plant breeders and other disciplines}

DNA marker technology, QTL theory and statistical methodology for QTL analysis have undergone rapid developments in the past two decades. These concepts and the jargon used by molecular biologists may not be clearly understood by plant breeders and other plant scientists [34]. In addition to this, many highly specialized pieces of equipment are based on sophisticated techniques used for molecular genotyping. Similarly, fundamental concepts in plant breeding may not be well understood by molecular biologists. This restricts the level of integration between conventional plant and molecular breeding and ultimately affects the development of new breeding lines [25].

\section{The discrepancy between marker development and marker application in breeding programs.}

There are far more publications on the development of markers than publications announcing the successful use of MAS in breeding. At first sight it is often difficult to distinguish whether a publication is actually reporting a MAS application or if only potential MAS applications of the actual research outputs are discussed. On closer inspection, most publications related to MAS deal with the detection/development of suitable markers and related research questions rather than reporting the application of MAS in breeding programs. 


\section{Marker technology development versus conversion into practical applications}

Although DNA markers have been available since the late 1980s, PCR-based markers allowing high throughput (microsatellite markers) became only available in the mid-to late 1990s. Only during the last five to ten years these markers have been widely used [34].

After the development of the first DNA-based markers Botstein et al. 1980 and after the term "marker-assisted selection" was first used by Beckmann \& Soller in 1986, it took another ten years until the first substantial article on the application of MAS in plant breeding was published Concibido et al. There seems to be a time lag of about ten years between the first application of new marker technologies and their widespread use in breeding programs. If this is the case, a notable increase in the number of publications describing MAS has to be expected in the next ten years and beyond Collard \& Mackill [34], especially if today's promises of SNP marker applications turn out to be true.

\section{Publication aspects}

QTL mapping - as a basic research process - regularly results in scientific publications. This explains the vast number of publications reporting the identification of new QTLs. However, scientists gain reputation mostly through carrying out innovative research and through publishing results within academic journals. Thus, there is little appeal to ensure that markers developed in research programs are also applied in breeding programs [34]. For plant breeding, in contrast, the aim is not to publish results but to release new varieties. Even if the new varieties are registered, details regarding the application of markers during the breeding process are not necessarily published.

In addition, in the private sector publication of results might even be discouraged due to competition reasons and the resulting unwillingness of researchers to share data and germplasm can cause serious limitation for the advancement of MAS applications.

\section{Lack of conversion of publications into practical applications}

A high proportion of published markers fails the translation step from research to application [4]. Converting promising publications into practical large-scale applications in breeding programs requires that different practical, economical, logistical, and genetical constraints are resolved. Before MAS realizes its full potential in public sector breeding programs,

a. Published markers need to be validated,

b. Simple, quick, and cheap technical protocols for tissue sampling need to be developed,

c. High throughput precision phenotyping systems for QTL mapping are needed and

d. Improved understanding of genotype by environment interaction and epistasis has to be gained [4].

\section{Genotype by environment interactions and effects of genetic background}

Genotype by environment interactions is complex phenomena which complicate the interpretation of genetical experiments and often make predictions difficult. They mostly cause difficulties in marker development and validation for quantitative traits. Ribaut \& Ragot 2006, having used a markerassisted backcross approach to improve drought adaptation in maize, state that the success of marker-selection for complex traits will largely depend on two things: the accuracy of plant phenotyping on the one hand and the understanding of genetic phenomena such as gene networks, epistasis, and GxE interactions on the other hand. Because quantitative traits are influenced by the environment they often show varied degrees of GxE interactions.

If quantitative traits are to be improved with MAS it is essential to have information about the GxE interactions. GxE interactions impede the repeatability of QTL mapping results and consequently reduce the efficiency of selection. It particularly becomes a problem where genotypes have to be selected in one environment and used in a different one. Especially QTLs with small effects can vary in magnitude and direction of effects, depending on environmental conditions. The extent of GxE interactions is not always known after conducting a mapping study, because such studies are usually restricted to a few years and/or locations [34]. Mostly modeling of the interactions is used to dissect the complex traits into manageable component traits and to describe the environmental effect on them. This supplies breeders with information containing less GxE interactions and therefore with QTLs that are more robust. Some scientists go as far as distinguishing between interactions of genotype and environment (GxE) and genotype and management (GxM), thus speaking of GxExM interactions. Many researchers consider management as part of the environment, but as management is that part of the environment that is manageable and therefore predictable to some extent, this can be a useful approach [3539].

Epistasis is the phenomenon that genes sometimes show a certain positive or negative effect only in combination with each other. For QTLs this can lead to unpredictability of expression in genetic backgrounds other than the one in which they have been detected. Where GxE interactions or epistasis are important, it is necessary to regularly re-estimate QTL effects within the breeding program, as suggested by Podlich et al. 2004.

\section{Economic aspects of marker application}

Only few studies compare the economical aspects of conventional phenotypic selection and MAS. Landmark papers are the one by Dreher et al. 2003 and the companion paper by Morris et al. 2003. Morris et al. 2003 state in their paper that "as most plant breeders well know, the cost of using DNA markers can vary greatly depending on the crop, the breeding application, the trait(s) being targeted, the availability of suitable marker 
technology, and other factors. This application specificity complicates economic analysis, but it does not invalidate it completely.

Well-designed case studies can help plant breeders make better decisions about choice of breeding strategy by generating detailed empirical information about the costs and time requirements of alternative selection methods." Morris et al. 2003 compared the costs for conventional and MAS methods for a particular breeding application. They introgressed an elite allele at a single dominant gene into an elite maize line and found that neither method shows clear superiority in terms of both cost and speed. Phenotypic selection schemes were less expensive, but MAS required less time. Thus, the decision for or against MAS is dependent on the availability of operating capital in the breeding program. Dreher et al. 2003 compared the cost-effectiveness of conventional and marker-assisted maize breeding. They used the conversion of lines into quality protein maize (QPM) as an example and the results indicate that the use of microsatellite markers was cost-effective compared to phenotypic selection. The authors conclude that such detailed budget analyses - if used with detailed empirical data from actual breeding programs - can be very useful to improve the efficiency of existing protocols and to make decisions about future technology choices. In general, the above studies show that no simple answer can be given to the question whether to apply MAS or phenotypic selection in a breeding program. But this type of analysis can nevertheless be very helpful if it is constantly updated as new genotyping systems become available [4].

\section{Intellectual property rights (IPR) in marker application}

In biotechnology there are many industrial applications with high economic value. Thus IPR - and especially patents - have become more and more important. The importance of molecular marker analyses for different applications was recognized very early, resulting in the filing of many patents in the last 10-15 years. From the private sector, there are no reports of the cost-benefit ratio associated with commercializing MAS-derived cultivars. However, the growing number of patent applications associated with MAS shows that the use of such approaches is seen as a comparative advantage in commercial breeding programs. If companies ensure their rights through the patenting of developed markers, no matter whether they are further utilized or not, they impede the use of these markers through others.

\section{Summary and Future Outlook}

Marker assisted selection (MAS) has already proven valuable for backcrossing of major genes into elite parents, using both foreground and background selection. This use is expected to increase as new genes and associated markers for economically important traits are identified. As an example of current opportunities for MAS in wheat, protocols for over 20 trait-associated markers are posted on the web site MAS Wheat: Bringing Genomics to the Wheat Fields (http://maswheat. ucdavis.edu/). Knowledge of actual gene sequences and design of PCR primers for specific alleles, will make MAS more powerful and informative across a range of genetic backgrounds.

More widespread use of MAS is also expected with the improvement of methods for marker analysis and identification of candidate genes for economic traits. The foreseeing economics will be a major driver of the application of MAS. For certain traits that are expensive or logistically difficult to evaluate, MAS is an attractive alternative. Time savings obtained through MAS may be as important as cost savings where there are competitive markets for improved cultivars. Any cost change in DNA extraction or genotyping methods, or on the other hand, in phenotypic evaluation methods, will affect the relative economic benefits of MAS (Figure 3).
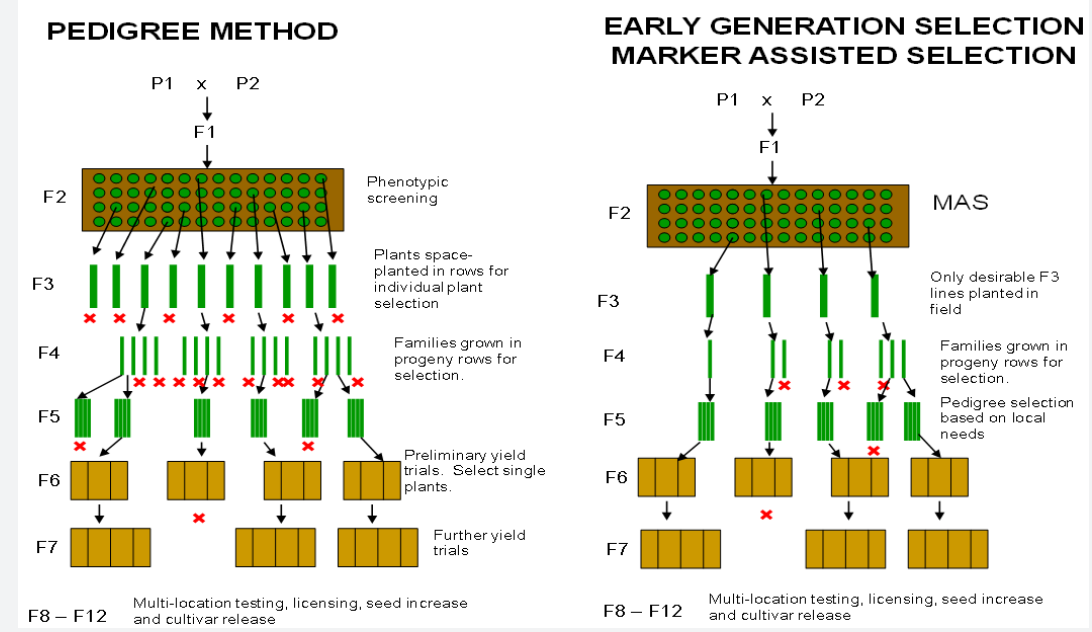

Figure 3: Early generation selection scheme (proposed by Ribaut \& Betran (1999).

In summary, MAS is a methodology that has already proved its value in some plant breeding situations. It is likely to become more valuable as a larger number of genes are identified and their functions and interactions elucidated. Reduced costs and optimized strategies for integrating MAS with phenotypic selection are needed before the technology can reach its full potential. 
Overall, marker assisted selection has proven to be a very useful technique in plant breeding. Through these techniques, plant breeders have been able to produce cultivars of agriculturally significant plants with genes for resistance to many diseases that were not possible before the advent of DNA technology.

\section{References}

1. Babu R, Nair SK, Prasanna BM, Gupta HS (2004) Integrating markerassisted selection in crop breeding: Prospects and challenges. Current Science 87(5): 607-619.

2. John R, Andrea S (2007) A marker-assisted selection as a tool for genetic improvement of crops, livestock, forestry and fish in developing countries: an overview of the issues. In: Elcio PG, et al. (Eds.), Markerassisted selection: Current status and future perspectives in crops, livestock, forestry and fish. Food and Agriculture Organization of the United Nations. Rome, Italy, pp. 3-14.

3. Yashitola J, Thirumurugan $\mathrm{T}$, Sundaram RM, Naseerullah MK, Ramesha MS, et al. (2002) Assessment of purity of rice hybrids using microsatellite and STS markers. Crop Sci 42(4): 1369-1373.

4. Xu Y, Beachell H, Mccouch SR (2004) A marker-based approach to broadening the genetic base of rice in the USA. Crop Science 44(6): 1947-1959.

5. Reif JC, Hamrit S, Heckenberger M, Schipprack W, Maurer HP, et al. (2005) Trends in genetic diversity among European maize cultivars and their parental components during the past 50 years. Theor Appl Genet 111(5): 838-845.

6. Liu SX, Anderson JA (2003) Marker assisted evaluation of Fusarium head blight resistant wheat germplasm. Crop Sci 43(3): 760-766.

7. McCartney CA, Somers DJ, Fedak G, Cao W (2004) Haplotype diversity at fusarium head blight resistance QTLs in wheat. Theor Appl Genet 109: 261-271.

8. Frisch M, Bohn M, Melchinger AE (1999) Comparison of selection strategies for marker-assisted backcrossing of a gene. Crop Sci 39: 1295-1301.

9. Tanksley SD, Young ND, Patterson AH, Bonierbale MW (1989) RFLP mapping in plant breeding: new tools for an old science. Bio/ Technology 7: 257-263.

10. Ragot M, Lee M (2007) Marker-Assisted Selection in Maize: Current status, potential, limitations and perspectives from the private and public sectors. In: Guimaraes EP et al. (Eds.), Marker-assisted Selection - Current Status and Future Perspectives in Crops, Livestock, Forestry and Fish. Rome, Italy, pp. 117-150.

11. Ribaut JM, Ragot M (2006) Marker-assisted selection to improve drought adaptation in maize: The backcross approach, perspectives, limitations, and alternatives. Journal of Experimental Botany 58(2): 351-360.

12. Mackill DJ (2006) Breeding for resistance to abiotic stresses in rice: the value of quantitative trait loci. In: Lamkey KR \& Lee M (Eds), Plant breeding: the Arnel R. Hallauer International Symposium, IA: Blackwell
Publication, Ames, USA, pp. 201-212.

13. Eathington SR, Crosbie TM, Edwards MD, Reiter RS, Bull JK (2007) Molecular markers in a commercial breeding program. Crop Science 47(Supplement 3): S154.

14. Peleman JD, van der Voort JR (2003) Breeding by design. Trends Plant Sci 8(7): 330-334.

15. Sanchez AC, Brar DS, Huang N, Li Z, Khush GS (2000) Sequence tagged site marker-assisted selection for three bacterial blight resistance genes in rice. Crop Sci 40: 792-797.

16. Walker D, Roger BH, All J, Parrott W (2002) Combining cry1Ac with QTL alleles from pi 229358 to improve soybean resistance to lepidopteran pests. Molecular breeding 9: 43-51.

17. Werner K, Friedt W, Ordon F (2005) Strategies for pyramiding resistance genes against the barley yellow mosaic virus complex (BaMMV, BaYMV, BaYMV-2). Mol Breed 16(1): 45-55.

18. Lande R, Thompson R (1990) Efficiency of marker-assisted selection in the improvement of quantitative traits. Genetics 124(3): 743-756.

19. Kuchel H, Fox R, Reinheimer J, Mosionek L, Willey N, et al. (2007) The successful application of a marker-assisted wheat breeding strategy. Molecular breeding 20: 295-308.

20. Bonnett DG, Rebetzke GJ, Spielmeyer W (2005) Strategies for efficient implementation of molecular markers in wheat breeding. Mol Breeding 15(1): 75-85.

21. Ribaut JM, Betran J (1999) Single large-scale marker-assisted selection (SLS-MAS). Mol Breed 5(6): 531-541.

22. Moreau L, Charcosset A, Gallais A (2004) Experimental evaluation of several cycles of marker-assisted selection in maize. Euphytica 137(1): 111-118.

23. Bohn M, Groh S, Khairallah MM, Hoisington DA, Utz HF, et al. (2001) Reevaluation of the prospects of marker-assisted selection for improving insect resistance against Diatraea spp. in tropical maize by cross validation and independent validation. Theor Appl Genet 103(6-7): 1059-1067.

24. Zhou WC, Kolb FL, Bai GH, Domier LL, Boze LK, et al. (2003) Validation of a major QTL for scab resistance with SSR markers and use of markerassisted selection in wheat. Plant Breed 122(1): 40-46.

25. Sharp PJ Johnston S, Brown G, McIntosh RA, Pallotta M, et al. (2001) Validation of molecular markers for wheat breeding. Aust J Agric Res 52: 1357-1366.

26. Han F, Romagosa I, Ullrich SE, Jones BL, Hayes PM, et al. (1997) Molecular marker-assisted selection for malting quality traits in barley. Mol Breed 3(6): 427-437.

27. Langridge P, Chalmers K (2005) The Principle: Identification and Application of Molecular Markers. In: Lörz H \& Wenzel G (Eds.), Molecular Marker Systems in Plant Breeding and Crop Improvement. Springer, Berlin, Germany, pp. 3-22.

28. Bretting PK, Widrlechner MP (1995) Genetic Markers and Plant Genetic Resource Management. Plant Breeding Reviews 13: 11-86. 
29. Sarah B, Maria RF (2011) Applications and potentials of marker assisted selection (MAS) in plant breeding. BfN-Skripten 298.

30. Kearsey MJ (1998) The principles of QTL analysis (a minimal mathematics approach). J Exp Bot 49: 1619-1623.

31. Kearsey MJ (1998) The principles of QTL analysis (a minimal mathematics approach). J Exp Bot 49: 1619-1623.

32. Melchinger AE, Utz HF, Schon CC (1998) Quantitative trait locus (QTL) mapping using different testers and independent population samples in maize reveals low power of QTL detection and large bias in estimates of QTL effects. Genetics 149(1): 383-403.

33. Li ZK, Yu SB, Lafitte HR, Huang N, Courtois B, et al. (2003) QTL by environment interactions in rice. I. Heading date and plant height. Theoret Appl Genet 108(1): 141-153.

34. Collard BCY, Jahufer MZZ, Brouwer JB, Pang ECK (2005) An introduction to markers, quantitative trait loci (QTL) mapping and marker-assisted selection for crop improvement: the basic concepts. Euphytica 142: 169-196.

35. David JM (2007) Molecular markers and marker-assisted selection in rice. In: Rajeev KV \& Roberto T (Eds.), Genomics-Assisted Crop Improvement: Genomics Applications in Crops 2: 169-186.

36. Marker assisted selection in wheat.

37. Langridge P, Lagudah E, Holton T, Appels R, Sharp P, et al. (2001) Trends in genetic and genome analyses in wheat: a review. Aust J Agric Res 52: 1043-1077.

38. Paterson AH, Lander ES, Hewitt JD, Peterson S, Lincoln SE, et al. (1988) Resolution of quantitative traits into Mendelian factors by using a complete linkage map of restriction fragment length polymorphisms. Nature 335(6192): 721-726.

39. Tanksley S (1993) Mapping polygenes. Ann Rev Genet 27: 205-233.

\section{Your next submission with Juniper Publishers} will reach you the below assets

- Quality Editorial service

- Swift Peer Review

- Reprints availability

- E-prints Service

- Manuscript Podcast for convenient understanding

- Global attainment for your research

- Manuscript accessibility in different formats

( Pdf, E-pub, Full Text, Audio)

- Unceasing customer service

Track the below URL for one-step submission https://juniperpublishers.com/online-submission.php 